\title{
Ten Eleven Translocation Gene 2 (TET2) Polymorphism in Acute Myeloid Leukemia
}

\author{
Nahla A. M. Hamed ${ }^{1 *}$, Ayda Aly Nazer ${ }^{1}$, Mona Wagdy Ayad ${ }^{2}$ and Tamer Sabah Sadiq ${ }^{1}$ \\ 'Hematology Unit, Department of Internal Medicine, Faculty of Medicine, Alexandria University, Egypt \\ ${ }^{2}$ Department of Clinical Pathology, Faculty of Medicine, Alexandria University, Egypt
}

\begin{abstract}
Background: Acute myeloid leukemia (AML) is a genetically heterogeneous disease in which somatic mutations that disturb cellular growth, proliferation, and differentiation accumulate in hematopoietic progenitor cells. TET methylcytosine dioxygenase 2 (TET2) gene mutations have been recognized in AML and appeared to have an adverse prognostic indicator in cytogenetically normal AML patients.

Methods: The study was designed to detect the TET2 single nucleotide polymorphism (SNP rs 6843141) in AML patients. Thirty one AML patients were enrolled in this study, median age was 41 years and male to female ratio was 1:1. Twenty five age and sex matched persons were considered a control group. Patients underwent thorough routine workup, Real time PCR for TET2 SNP polymorphism expression with an ABI Prism Genamp 7500 using peripheral blood c-25767872-10, rs 6843141 (UK) was done for all cases and controls.

Result: TET2 polymorphism SNP rs 6843141 was detected in all AML cases with $26(83.9 \%)$ patients showing homozygous pattern and 5 (16.1\%) patients showing heterozygous pattern while the control group was mostly wild type (11 patients, $44 \%$ ) and heterozygous group (11 patients, $44 \%$ ), $\mathrm{p}<0.001$. The highest homozygous pattern (19 out of 26 patients, $73 \%$ ) was present in AML M1, M2 and M5 subtypes, which are the commonest encountered subtypes among our cases. Fifteen out of 27 AML patients were cytogenetically normal (55.5\%). TET2 polymorphism showed higher bone marrow blast percentage among homozygous group, $\mathrm{p}=0.015$.
\end{abstract}

Conclusion: TET2 SNP rs 6843141 polymorphism is common among Egyptian AML cases irrespective of their cytogenetic abnormality.

Keywords: Acute Myeloid Leukemia, TET2, Gene Mutations, Polymorphism

\section{Introduction}

Acute myelogenous leukemia (AML) is a heterogeneous group of disease characterized by uncontrolled proliferation of myeloid progenitor cells that gradually replace normal hematopoiesis in the bone marrow. ${ }^{(1)}$ The genetic changes arising in the neoplastic clone lead to cascades of molecular events that cause abnormal proliferation, aberrant differentiation, and inhibition of normal hematopoiesis by the malignant cells. ${ }^{(2-4)}$

Characterization of transforming genetic events is increasingly important in establishing diagnosis, defining prognosis, and planning therapy in AML.(5) The current challenge is to understand the molecular mechanisms of AML and design leukemia- specific treatments effective in chemotherapy- resistant disease and applicable to older patients. ${ }^{(2)}$

TET enzymes are one of the homeostatic links between intracellular metabolism and epigenetic gene regulation.

(6) The mammalian TET family contains three members, TET-1,TET-2 and TET-3, ${ }^{(7)}$ of them TET-2 has pleiotropic roles during hematopoiesis, including stem cells selfrenewal, lineage commitment, and terminal differentiation of monocytes. ${ }^{(6)}$ Homozygous and heterozygous mutations in TET-2 gene are recurrent events in human hematopoietic malignancies. Most of these mutations decrease TET-2 enzymatic activity by truncating the protein or affecting its catalytic activity. TET-2 deletion is sufficient to initiate myeloid transformation. It is appealing to assume that loss of a TET protein could contribute to the gene-specific hypermethylation that is often observed in cancer. Thus, TET-2 haploinsufficiency, may be sufficient to change the properties of HSCs. ${ }^{(6)}$ TET2 mutations appeared to be an adverse prognostic indicator in cytogenetically normal AML patients. ${ }^{(8)}$

\section{Materials and Methods:}

Thirty one AML patients were enrolled in the study with a median age of 41years and male to female ratio of $1: 1$, in addition to 25 persons matched for age and gender are considered a control group. Patients underwent thorough history taking, physical examination and routine investigations along with bone marrow aspirate, flow cytometry, and cytogenetics. DNA extraction and Real time PCR for TET2 SNP polymorphism with an ABI Prism GeneAmp 7500 using peripheral blood c-25767872-10, rs 6843141, lot p161221-001 H07 PN*40 (manufactured in UK) was assessed in all patients and controls. 


\section{Result}

Tables 1 show demographic data, and Tet2 polymorphism of the cases and control groups. Both groups were age and gender matched (p: 0.811 and 0.832 respectively). TET2 polymorphism SNP rs 6843141 was detected in all AML cases with $26(83.9 \%)$ patients showing homozygous pattern and $5(16.1 \%)$ patients showing heterozygous pattern while the control group was mostly wild type (11 patients, $44 \%$ ) and heterozygous group (11 patients, $44 \%), \mathrm{p}<0.001$. The highest homozygous pattern (19 out of 26 patients, 73\%) was present in AML M1, M2 and M5 subtypes (table 2 and 3). Fifteen out of 27 AML patients were cytogenetically normal $(55.5 \%)$. There is no relation between TET2 polymorphism and clinical presentation, organ infiltration, $\mathrm{CBC}$, bone marrow blasts or cytogenetics, $\mathrm{p}>0.05$. Tet2 polymorphism showed higher bone marrow blast percentage among homozygous than heterozygous patients, $p=0.015$ (table 2). Tet2 polymorphism did not affect the treatment outcome of patients, $\mathrm{p}>0.05$ (data not shown).

Table 1: biological characteristics, Complete Blood Count and Tet2 polymorphism in the studied groups.

\begin{tabular}{|c|c|c|c|c|c|}
\hline Parameter & \multicolumn{2}{|c|}{ AML cases $(n=31)$} & \multicolumn{2}{|c|}{ Control group $(n=25)$} & P-value \\
\hline \multicolumn{5}{|c|}{ Age (years) } & \\
\hline Mean \pm SD & \multicolumn{2}{|c|}{$43.7 \pm 14.3$} & \multicolumn{2}{|c|}{$42.0 \pm 8.2$} & \multirow[t]{4}{*}{$P=0.811$} \\
\hline Median & \multicolumn{2}{|c|}{41.0} & \multicolumn{2}{|c|}{41.0} & \\
\hline Minimum- maximum & \multicolumn{2}{|c|}{$22-73$} & \multicolumn{2}{|c|}{$28-59$} & \\
\hline Gender & No. & $\%$ & No. & $\%$ & \\
\hline Male & 17 & 54.8 & 13 & 52.0 & \multirow[t]{2}{*}{$P=0.832$} \\
\hline Female & 14 & 45.2 & 12 & 48.0 & \\
\hline TeT2 polymorphism & No. & $\%$ & No. & $\%$ & \\
\hline Wild type & 0 & 0.0 & 11 & 44.0 & \multirow[t]{3}{*}{$P=<0.001^{*}$} \\
\hline Homozygous type & 26 & 83.9 & 3 & 12.0 & \\
\hline Heterozygous type & 5 & 16.1 & 11 & 44.0 & \\
\hline
\end{tabular}

Table 2: comparison between homozygous and heterozygous cases as regards bone marrow blasts (\%) and FAB classification.
Laboratory investigations
Homozygous group $(n=26)$
Heterozygous group $(n=5)$
P-value

Bone marrow blasts (\%)

\begin{tabular}{|c|c|c|c|c|c|}
\hline Mean \pm SD & \multicolumn{2}{|c|}{$68.0 \pm 22.9$} & \multicolumn{2}{|c|}{$39.6 \pm 15.1$} & \multirow{4}{*}{$P=0.015^{*}$} \\
\hline Median & \multicolumn{2}{|c|}{77.0} & \multicolumn{2}{|c|}{36.0} & \\
\hline Minimum- maximum & \multicolumn{2}{|c|}{ 10- 90} & \multicolumn{2}{|c|}{$25-60$} & \\
\hline FAB classification & No. & $\%$ & No. & $\%$ & \\
\hline M0 & 4 & 15.4 & 0 & 0.0 & \multirow{6}{*}{$P=0.020^{*}$} \\
\hline M1 & 6 & 23.1 & 1 & 20.0 & \\
\hline M2 & 7 & 26.9 & 0 & 0.0 & \\
\hline M3 & 0 & 0.0 & 2 & 40.0 & \\
\hline M4 & 3 & 11.5 & 0 & 0.0 & \\
\hline M5 & 6 & 23.1 & 2 & 40.0 & \\
\hline
\end{tabular}


Table 3: Bone Marrow Blasts, Cytogenetics and Tet2 Polymorphism of M1, M2 and M5 AML patients.

\begin{tabular}{|c|c|c|c|c|c|c|c|}
\hline Parameter & \multicolumn{2}{|c|}{ M1 AML $(n=7)$} & \multicolumn{2}{|c|}{ M2 AML $(n=7)$} & \multicolumn{2}{|c|}{ M5 AML $(n=8)$} & P-value \\
\hline \multicolumn{7}{|c|}{ Bone marrow blasts (\%) } & \\
\hline Mean \pm SD & \multicolumn{2}{|c|}{$67.3 \pm 21.8$} & \multicolumn{2}{|c|}{$56.6 \pm 29.0$} & \multicolumn{2}{|c|}{$69.5 \pm 25.1$} & \multirow{3}{*}{$P=0.519$} \\
\hline Median & \multicolumn{2}{|c|}{66.0} & \multicolumn{2}{|c|}{63.0} & \multicolumn{2}{|c|}{80.0} & \\
\hline Minimum- maximum & \multicolumn{2}{|c|}{$32-90$} & \multicolumn{2}{|c|}{ 10- 88} & \multicolumn{2}{|c|}{ 25- 90} & \\
\hline TET2 polymorphism & No. & $\%$ & No. & $\%$ & No. & $\%$ & \\
\hline Homozygous type & 6 & 85.7 & 7 & 100.0 & 6 & 75.0 & \multirow[t]{2}{*}{$P=0.743$} \\
\hline Heterozygous type & 1 & 14.3 & 0 & 0.0 & 2 & 25.0 & \\
\hline Cytogenetics $(n=20) \#$ & No. & $\%$ & No. & $\%$ & No. & $\%$ & \\
\hline Normal & 4 & 66.7 & 3 & 50.0 & 6 & 75.0 & \multirow[t]{2}{*}{$P=0.842$} \\
\hline Abnormal & 2 & 33.3 & 3 & 50.0 & 2 & 25.0 & \\
\hline
\end{tabular}

Significant at $P \leq 0.05$, \# Two samples faced technical problems

\section{Discussion}

The AML patients group had a median age of 41 years. De novo AML was $29(93.5 \%)$ of cases and only $2(6.5 \%)$ cases were secondary (CML in blastic crisis). This was similar to that reported by Hamed et al ${ }^{(9)}$ where the median age of the studied Egyptian AML cases was 40 years old and most patients were de-novo cases (87.3\%). In the same study, TET2 gene expression showed under-expression of TET2 gene that was present in $90.6 \%(0.3098 \pm 0.3846)$ of 33 studied AML patients when assessed by real-time PCR. This could be attributed to several factors including a mutation, or polymorphism patterns. ${ }^{(9)}$

In our patients, AML patients showed homozygous $(83.9 \%)$ and heterozygous (16.1\%) expression of TET2 gene polymorphism while controls showed a wild type (44\%) and heterozygous (44\%) expression polymorphism. On contrary, Li et al ${ }^{(10)}$ in 2016 estimated the prevalence of TET2 mutations in their studied children AML patients to be $1.4 \%$. The most common polymorphism was I $1762 \mathrm{~V}$ (45\%), followed by V218M (12\%), P29R (6\%), and F868L $(6 \%)$. Kutny et al ${ }^{(11)}$ reported in 2015 that the TET2 SNP rs 6843141 was detected in $11.63 \%$ of children with AML. In the present study, patients were categorized according to pattern of SNP rs 6843141 polymorphism expression as wild type, homozygous and heterozygous expression. It was noted that $83.9 \%$ of our patients showed homozygous TET-2 gene expression.

TET-2 (mut) are characterized by a weak gene-expression pattern, which, nevertheless, reflected TET-2 (mut) associated biology. ${ }^{(12)}$ This is usually followed by next generation DNA sequencing for TET-2 to detect the landscape of abnormalities in the gene sequence. ${ }^{(13)}$
Comparing homozygous versus heterozygous polymorphism as regard clinical presentations and $\mathrm{CBC}$ findings (including peripheral blast \%) of patients showed non statistically significant difference $(p>0.05)$. in our cases, anemia was noted in $15.4 \%$ among homozygous versus $20.0 \%$ among heterozygous, while bleeding was present in $42.3 \%$ versus $80.0 \%$ and infection in $84.6 \%$ versus $80.0 \%$ respectively. This differed from that reported by Metzeler et al ${ }^{(14)}$ who reported that TET-2 mutations were associated with higher pretreatment white blood cell counts $(\mathrm{p}=0.04)$. Chou et al ${ }^{(15)}$ and Nibourel et $\mathrm{al}^{\left({ }^{(16)} \text { reported }\right.}$ no relation between TET2 mutation and $\mathrm{Hb}, \mathrm{WBCs}$ and platelets. TET-2 gene mutations accumulate with age in otherwise healthy subjects with normal hematopoiesis. ${ }^{(17)}$

Normal cytogenetic was present in $54.5 \%$ of homozygous and $60.0 \%$ among heterozygous group. Cytogenetic abnormalities in homozygous patients included $\mathrm{t}(8: 21)$ in 2 cases, trisomy $8+$ in 2 cases, $\mathrm{t}(16: 16)$ in 2 cases, $\mathrm{t}(3: 3)$ in 1 case, inv 16 in 1 case, trisomy $7+$ in 1 cases, $t(9: 22)$ in 1 case whereas the 2 cases with $t(15: 17)$ were heterozygous. This is similar to that reported by Schnittger et al ${ }^{(18)}$ where normal karyotyping constituted $55.9 \%$ of cases $(311 / 556)$.

As regards the bone marrow blast percent, homozygous cases showed statistically significant higher mean blast \% 68.0 22.9 compared to $39.6 \pm 15.1$ among heterozygous group. The highest homozygous pattern (19 out of 26 patients, $73 \%$ ) was present in AML M1, M2 and M5 subtypes, which are the commonest encountered subtypes among our cases.TET-2 is important for normal myelopoiesis, and disruption of TET-2 enzymatic activity may favor myeloid tumorigenesis. ${ }^{(19)}$ 
TET-2 mutations were associated with deregulation of genes involved in stem-cell self-renewal, cell cycle control, and cytokine and growth factor signalling, which may help explain their adverse prognostic impact in previous study. ${ }^{(14)}$ Neither homozygous nor heterozygous TET-2 polymorphism expression affected response to therapy in our studied cases $(\mathrm{p}>0.05)$. TET-2-mutated patients had lower complete remission rate $(\mathrm{CR} p=0.007)$, shorter event free survival $(\mathrm{p}=<0.001)$, shorter disease free survival (DFS, $p=0.003)$, and shorter overall survival $(p=0.001)$ in Metzeler et al ${ }^{(14)}$ study. Yet, it differed from that reported by Schlenk et al. ${ }^{(20)}$ Abdel-Wahab et al $^{(21)}$ reported that TET-2 mut were found in $12 \%$ of patients with AML and were associated with decreased overall survival.

TET2 probably plays a role in the pathogenesis and progression of AML. Epigenetic modifying genes (DNMT3a, TET-2 and IDH1/2) are important in control of methylation. These mutations are found at higher frequency in conjunction with other mutations supporting the hypothesis of genetically unstable leukemic cells requiring numerous mutations and chromosomal abnormalities. As the mutations of epigenetics modifying genes does not differ between pretreatment and relapse samples, they may be used as minimal residual disease marker. ${ }^{(22)}$ Besides, TET-2 mutations were reported as an independent predictor of a higher overall response rate to azacitidine, in patients with low blast count AML. ${ }^{(23)}$ No reports were available for the effect of TET-2 mutations on response to allogeneic peripheral hematopoietic stem cell transplantation.

\section{Conclusion}

From this study we concluded that TET2 SNP rs 6843141 polymorphism is common among Egyptian AML cases irrespective of their cytogenetic abnormality. Further studies on larger and more homogenous patient populations are needed to evaluate the prognostic impact of TET2 mutations in AML and to assess the predictive role of TET2 expression in response to hypomethylating agents.

\section{Acknowledgements}

We would like to thank all the hematology department, the medical genetics center and clinical pathology whom participation have brought out this research and whose valuable effort was most efficient.

\section{Reference}

1. Smith BD, Sung L. Acute myeloid leukemia. blood 2013;5(18):481-9.

2. Suhag VS, Malkovska V, Solomon SR. Acute myeloid leukemia.. In: Griffin P. Rodgers and Neal S. Young (eds). Bethesda Handbook of Clinical Hematology. Lippincott: Williams \& Wilkins 2013;131-47.
3. DolnikA, Engelmann JC, Scharfenberger-Schmeer M, Mauch J, Kelkenberg-Schade S, Haldemann B, et al. Commonly altered genomic regions in acute myeloid leukemia are enriched for somatic mutations involved in chromatin remodeling and splicing. Blood 2012;120(18):e83-e92.

4. Marcucci G, Haferlach T, Dohner H. Molecular genetics of adult acute myeloid leukemia: prognostic and therapeutic implications. J Clin Oncol 2011;29(5):475-86.

5. Appelbaum FR, Gundacker H, Head DR, Slovak ML, Willman CL, Godwin JE, et al. Age and acute myeloid leukemia. Blood 2006;107(9):3481-5.

6. Solary E, Bernard O, Tefferi A, Fuks F, Vainchenker W. The Ten-Eleven Translocation-2 (TET2) gene in hematopoiesis and hematopoietic diseases. Leukemia 2014;28(3):485-96.

7. Tan L, Shi YG. Tet family proteins and 5-hydroxymethylcytosine in development and disease. Development 2012;139:1895-902.

8. Liu W-J, Tan X-H, Luo X-P, Guo B-P, Wei Z-J, Ke Q, et al. Prognostic significance of Tet methylcytosine dioxygenase 2 (TET2) gene mutations in adult patients with acute myeloid leukemia: a meta-analysis. Leukemia \& lymphoma 2014;55(12):2691-8.

9. Hamed N, El Halawani N, Kassem H, Ayad M, Dammag E. TET2 expression in a cohort of Egyptian acute myeloid leukemia patients. The Egyptian Journal of Haematology 2015;40(4):159-65.

10. Li M-J, Yang Y-L, Lee N-C, Jou S-T, Lu M-Y, Chang H-H, et al. Tet oncogene family member 2 gene alterations in childhood acute myeloid leukemia. Journal of the Formosan Medical Association 2016;115(9):801-6.

11. Kutny MA, Alonzo TA, Gamazon ER, Gerbing RB, Geraghty D, Lange B, et al. Ethnic variation of TET2 SNP rs2454206 and association with clinical outcome in childhood AML: a report from the Children/'s Oncology Group. Leukemia 2015;29(12):2424-6.

12. Gaidzik VI, Paschka P, Spath D, Habdank M, Kohne $\mathrm{CH}$, Germing $\mathrm{U}$, et al. TET2 mutations in acute myeloid leukemia (AML): results from a comprehensive genetic and clinical analysis of the AML study group. J Clin Oncol 2012;30(12):1350-7.

13. Weissmann S, Alpermann T, Grossmann V, Kowarsch A, Nadarajah N, Eder C, et al. Landscape of TET2 mutations in acute myeloid leukemia. Leukemia 2011;26:934-42.

14. Metzeler KH, Maharry K, Radmacher MD, Mrózek K, Margeson D, Becker $\mathrm{H}$, et al. TET2 mutations improve the new European LeukemiaNet risk classification of acute myeloid leukemia: A Cancer and Leukemia Group B Study. J Clin Oncol 2011;29(10):1373-81.

15. Chou W-C, Chou S-C, Liu C-Y, Chen C-Y, Hou H-A, Kuo $\mathrm{Y}-\mathrm{Y}$, et al. TET2 mutation is an unfavorable prognostic factor in acute myeloid leukemia patients with intermediaterisk cytogenetics. Blood 2011;118(14):3803-10.

16. Nibourel O, Kosmider O, Cheok M, Boissel N, Renneville A, Philippe N, et al. Incidence and prognostic value of TET2 
alterations in de novo acute myeloid leukemia achieving complete remission. Blood 2010;116(7):1132-5.

17. Solary E, Bernard OA, Tefferi A, Fuks F, Vainchenker W. The Ten-Eleven Translocation-2 (TET2) gene in hematopoiesis and hematopoietic diseases. Leukemia 2014;28(3):485-96.

18. Schnittger S, Nadarajah N, Alpermann T, Eder C, Kohlmann A, Kuznia S, et al. Comparison of mutation patterns between diagnosis and relapse in 556 adult patients with AML shows high variability of stability. Blood 2013;122(21):4978.

19. Ko M, Huang Y, Jankowska AM, Pape UJ, Tahiliani $\mathrm{M}$, Bandukwala HS, et al. Impaired hydroxylation of 5-methylcytosine in myeloid cancers with mutant TET2. Nature 2010;468(7325):839-43.

20. Schlenk RF, Dohner K, Mack S, Stoppel M, Kiraly F, Gotze $\mathrm{K}$, et al. Prospective evaluation of allogeneic hematopoietic stem-cell transplantation from matched related and matched unrelated donors in younger adults with high-risk acute myeloid leukemia: German-Austrian trial AMLHD98A. J Clin Oncol 2010;28(30):4642-8.

21. Abdel-Wahab O, Mullally A, Hedvat C, Garcia-Manero G, Patel J, Wadleigh M, et al. Genetic characterization of TET1, TET2, and TET3 alterations in myeloid malignancies. Blood 2009;114(1):144-7.

22. Wakita S, Yamaguchi H, Omori I, Terada K, Ueda T, Manabe E, et al. Mutations of the epigenetics-modifying gene (DNMT3a, TET2, IDH1/2) at diagnosis may induce FLT3-ITD at relapse in de novo acute myeloid leukemia. Leukemia 2013;27(5):1044-52.

23. Itzykson R, Kosmider O, Cluzeau T, De Mas V, Dreyfus F, Quesnel B, et al. Presence of TET2 mutation predicts a higher response rate to azacitidine in MDS and AML post MDS. ASH Annual Meeting Abstracts 2010;116(21):439.

*Corresponding author:

Professor Nahla Abdel Moneim Hamed, Postal Address: faculty of Medicine, Alexandria University, Alexandria, Egypt

Phone: +91 00201223507914

Email: drhamedn@hotmail.com

Financial or other Competing Interests: None.

Annals of Advanced Medical Science, Vol. 2; Issue 2, April-June, 2018 